\title{
A Research on key Vision Technologies of Red Revolution site in Gannan Soviet area based on UE4 Virtual engine platform
}

\author{
Zhiqiang Wang ${ }^{1, *}$ Dandan $\mathrm{Li}^{1} \quad$ Tingting Fan ${ }^{1}$ \\ ${ }^{1}$ Jiangxi University of Technology Ganzhou, Jiangxi 341000 ,China
}

\begin{abstract}
The Red Revolution site in Gannan Soviet area is an important cultural heritage of China, which is represented by Yeping and Shazhouba in Ruijin City, and has important spiritual and cultural value. The red revolution site in Gannan Soviet area is taken as the object of study from the point of view of protection and inheritance. And the visualization technology is studied under the platform of UE4 virtual engine combined with three-dimensional digital technology. In addition, the core technologies such as the model making of 3D Max software, the material construction of UE4 virtual platform and the interface interaction realization of UE4 virtual platform in the visualization technology of red revolution site in Gannan Soviet area are studied. They are all studied by means of field investigation, data measurement and quantitative experiments.At the same time, this paper points out the ways, methods and strategies of realization from the level of visualization technology, and its object characteristic is to combine the revolutionary site with the paper. This also provides a technical guarantee for the development of Red Revolution sites and other similar visualization projects in Gannan Soviet area.
\end{abstract}

\section{Introduction}

With the rapid development of digital technology, the ability of people to visualize visible and invisible cultural sites has been gradually strengthened. It makes the audio-visual experience into an advanced stage, and the development of UE4 virtual engine promotes the visualization field. Many games are developed through the UE4 virtual engine, such as PUBG: exciting Battlefield, Game for Peace and so on. These games in the beautiful screen, immersive experience, so that users get an unprecedented experience, but they are also recognized by everyone.Nowadays we are faced with all kinds of cultures, especially when facing the extinction of culture. At this time, how to rescue and protect them has become an important proposition for all countries to think about.As an important cultural site left by our country in the period of modern revolution, the revolutionary site group in Gannan Soviet area is of farreaching significance among these cultures. However, due to its remote location, lack of economic conditions, natural climate and man-made destruction and other factors, it will also face the fate of collapse.Therefore, it is of great significance to carry out visualization technology research on the revolutionary site group in Gannan Soviet area which is from the point of view of digital protection and inheritance; using UE4 virtual engine platform; combined with relevant threedimensional technology.

\section{The Feasibility Analysis of Visualization of Red Revolution sites in Gannan Soviet Area}

The ruins of the Red Revolution in the Gannan Soviet area are mainly located in Yeping and Shazhouba in Ruijin City. There are more than 40 sites, including the site of the first National Congress of the Chinese Soviet Union, the site of the General Office, the site of the allChina Federation of Trade unions, the former site of Lenin Primary School, and so on.The Red Revolution site in Gannan Soviet area is an important cultural heritage of China. Although it was strongly protected and supported by the government and set up a corresponding management unit for it, it also suffered irreversible damage due to time and natural corrosion and other factors.And Gannan is an important area of Chinese modern revolution, where red revolution sites are widely distributed. However, the protection manpower and financial resources here are limited, which has led to the accelerated extinction of many unnoticed and less famous red sites.Therefore, the visualization form of digital technology has the characteristics of authenticity, science and rigor from the point of view of protection and inheritance. And it is convenient for users to experience and spread online.

The overall division of the red revolution sites in Gannan Soviet area represented by Yeping and Shazhouba in Ruijin City is standardized. The modeling structure of its architectural sites is clear, and the data are well preserved, which is conducive to the

Corresponding author: Zhiqiang Wang wanto126@126.com 
visualization of digital technology. In the choice of visualization technology, there have been many visualization development tools in the software in recent years, such as OpenGL, Maya, Unreal Engine, Paradigm and so on. And the UE4 virtual engine has the most technical advantages in the visual performance of architectural sites among them.. On the one hand, UE4 can create high-quality picture quality in real time, shorten the time of high-quality rendering, and feedback the picture effect to the designer in time.On the other hand, UE4 can access the data of other software, such as 3D Max, Maya or C4DMagneUE4 can summarize and organize the data of the completed 3D model. At the same time, the output of UE4 virtual engine is rich in picture and video formats, which can meet the needs of the current market.

Due to the visualization of the Red Revolution site in the Gannan Soviet area of the UE4 virtual platform, it has dual social benefits for the site itself and the local culture. On the one hand, the visualization project under the UE4 virtual platform will be an open system, which can modify the data parameters with the change of the revolutionary sites, so as to ensure the authenticity and validity of the visualization object. This open mode of visual provides a scientific and reasonable way to protect tangible cultural sites. On the other hand, the establishment of the visualization project in digital form can facilitate the two-way online and offline experience and dissemination of the virtual red revolution sites in Gannan Soviet area. It has the characteristics of digitality, immediacy and sharing, such as organizing young students from all over the country to visit revolutionary sites online; feel the spiritual content of red culture; and gain a deeper understanding of the hard-won new China [2].

\section{The Visualization technologies of three- dimensional model and material of Red Revolution site in Gannan Soviet Area.}

\subsection{The Making of 3D Model for Visualization of Red Revolutionary site in Gannan Soviet Area}

The making of visual 3D model mainly depends on the relevant $3 \mathrm{D}$ software such as $3 \mathrm{ds}$ max, $3 \mathrm{ds}$ maya and so on. And the $3 \mathrm{~d}$ s max software is the current mainstream 3D making software. It is often used in the 3D template production of visualization projects, which has the advantages of professional, convenient and flexible.

Therefore, the polygon modeling module of $3 \mathrm{ds} \max$ software will be conducive to the concrete completion of the visual three-dimensional model stage of the Red Revolution site in the Soviet area of southern Jiangxi in view of the complex geographical form and many building volumes .

From the perspective of the macroscopic number of buildings at the Red Revolution site in the Soviet area of southern Jiangxi, the visualization site covers an area of about 330000 square meters in Yeping and Shazhouba, and there are only more than 40 single sites. The creation of modeling needs to obtain accurate structural data or image morphology of Soviet revolutionary sites under the visual three-dimensional model production of $3 \mathrm{ds}$ max software. For example, the structural data and image shape were mainly completed by surveying and photographing in the making of the three-dimensional model of the former site of the first Soviet Congress (Xie Family ancestral Hall). In addition, the shooting of the structural image of the site is mainly through the on-site return and fixed-point patrol of DJI Mavic Air to obtain the $4 \mathrm{~K}$ high-definition image structure of the site to meet the needs of model production, as shown in figure 1 .

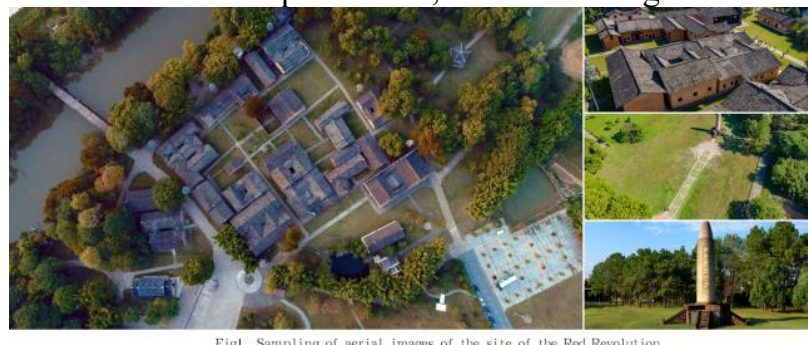

In the method of making the three-dimensional model of the Red Revolution site in Gannan Soviet area. On the one hand, we think of the convergence of the later UE4 virtual engine platform, so we use the default centimeter unit size in UE4; on the other hand, in the modeling process of 3D Max software, the specific model needs to conform to the topology law of the model UV. And we try our best to avoid the situation that three sides occupy one sideline together, and let alone multiple faces overlap to ensure the rationality of the $3 \mathrm{D}$ model wiring splitting process, as shown in figure2.

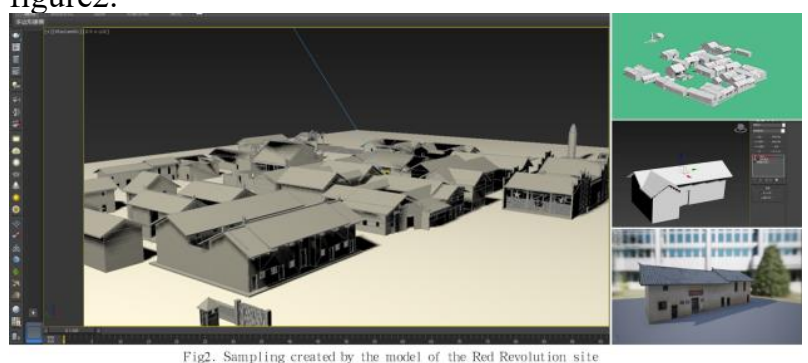

3D model UV split production is an important transition link In the process of visualization. And it connects the two stages of the model and the material, but also affects the quality and efficiency of the material stage [4]. Three-dimensional model UV splitting is usually completed in two ways, the first one is completed by using relevant independent UV split plugins, such as UVLayout, UnwrapPro, etc., and the second one is made by the inherent UV split module in 3D software, such as the UV splitter in 3D Max and Maya software. The UV split is affected by the overall wiring and complicated details of each site model on the threedimensional model of the Red Revolution site in the Soviet area of southern Jiangxi. Therefore, we think from experience that the UV splitting of the threedimensional model of the Red Revolutionary site in Gannan Soviet area mainly needs to pay attention to the following aspects: on the one hand, to avoid the overlap and triviality of UV in the split, and it is not easy to draw the map later, which affects the mapping effect in the 
later stage of the model; on the other hand, it is to smooth and stretch the seriously distorted model as far as possible. Because UV not only affects the texture map, but also has an important impact on the normal, bump, displacement and other related maps. Finally, in the UV texture integration, we need to make reasonable use of UV space and put the model map on the same UV map as far as possible. In this way, we can save time by avoiding forgetting the location and size of the map when filling the map in PS.

\subsection{The Visual 3D Material Making of Red Revolutionary Site in Gannan Soviet Area}

Material making is an important part of threedimensional visualization process, which involves the quality of the overall visualization project and the sensory effect of users. In the making environment of the UE4 virtual engine platform, the material module has been separated from other three-dimensional software and separately integrated into the architecture of the platform. It has the characteristics of integration and high efficiency [5]. The making of UE4 material needs to go through three stages: material map collection, map processing and UE4 material performance, which is taking the former site of the first Soviet Congress as an example.In addition, in order to enhance the reality of the three-dimensional virtual world of the site visualization, we have carried on the map collection, but also need to shoot the sub-structure image of the site site. In the shooting process, the map quality needs to be combined with weather, light and other factors for pointto-point analysis and collection, so as to obtain a highly clear and color-saturated pattern. In the mapping processing, firstly we need to refer to the Uv split map of the model in the Adobe Photoshop software to set the corresponding basic color, and adjust the color difference of some maps, so as to achieve the unity of the map color; secondly, we will use Materialize software to make roughness, normal, AO and other material maps. After the above phase is completed, the completed map will be imported into the UE4 steal virtual engine to make the corresponding shader. Then we use the created base shader to connect the map with the corresponding channel on the basis of modifying the parameters [6].We combine the real effects and artistic processing of the site to add nodes to adjust the parameters of each map to make the shader the best visual effect, as shown in figure 3 .

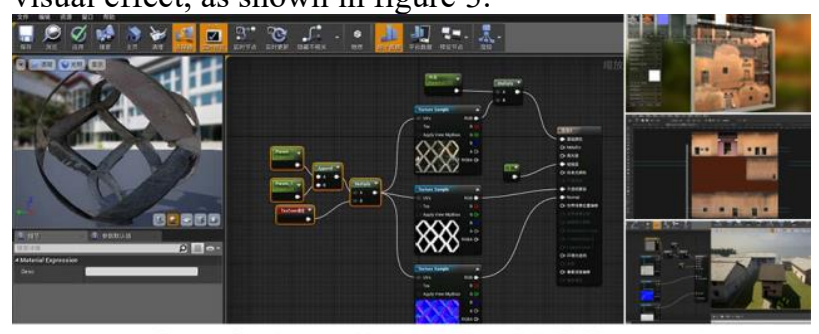

Figure3. Samples created by the materials of the Red Revolution site

\section{The UE4 Visual interaction and Interface Design of Red Revolution site in Gannan Soviet area}

Compared with many virtual platform technologies, the visual interactive interface implementation technology of UE4 virtual engine platform has many advantages. It is mainly reflected in good interaction, strong sense of experience, especially paying attention to man-machine interactive feedback. From the perspective of game experience, all kinds of online games developed by UE4 virtual engine platform can better meet the interactive experience of users at all stages, because it pays attention to the connotation and style of game interface design [7]. Therefore, under the UE4 virtual engine platform, the visual interaction and interface design of the Red Revolution site in Gannan Soviet area need to be deeply developed and designed according to their own cultural connotations and characteristics.

\subsection{UI Design of Interface}

In the application of the game or virtual simulation industry, the user interface design is to convey the equipment information of the UI interface or subinterface of the whole game system to the user accurately and reasonably. For the UI interface design of the Red Revolution site in the Soviet area, we mainly analyzed the interface visualization of the Red Revolution site in the Soviet area.UI needs to contact the ontology content of the visual simulation project and convey the information to the reader. In other words, we need to summarize and design the contents related to the ruins of the Red Revolution.For example, in the visual interaction of the red revolution sites, the interface setting can design the interface image with red cultural characteristics around the revolutionary figures of the red revolution in the Gannan Soviet area. From the perspective of interactive experience, we can start from the group objects and cultural literacy in order to achieve the purpose of human-computer interaction, and take the revolutionary sites in Gannan Soviet area as the main research object. And we should pay attention to the overall tone of the whole interface UI and the typesetting design of the whole graphics [9]. In addition, the design idea can absorb the red history and cultural heritage of the revolutionary sites in Gannan Soviet area on the specific UI icon. For example, the elements extracted from Soviet clothing and historical objects are designed for the icons of the interface.

\subsection{Design of interactive function}

The design of interaction function plays an important role in the interaction design of the whole visual project. In our visualization research project of the Red Revolution site in Gannan Soviet area, how to design the overall interaction of the regional culture project? And how to achieve a sense of distance in the user's interactive experience? At the same time, in the specific setting of the interaction, the setting of the virtual area of 
the interactive function requires the experimental experience of the man-machine. It integrates the elements of revolutionary culture in the Soviet area into the design of this interactive function, and makes culture and design support each other. And the fusion design of virtual characters and interactive functions is the current trend of visual performance [10].In the process of building virtual characters in virtual reality, on the one hand, we can enable users to guide characters and set different information levels by clicking on the screen, and to understand and answer questions about the red culture of the Soviet area. It not only helps people understand the culture of the Red Revolutionary Base in Gannan Soviet area, but also enhances the interesting experience of users. On the other hand, we can make users look for knowledge fragments in the process of experience by means of jigsaw puzzles. In this process, we can make users have a better understanding of the revolutionary sites in the Soviet area as a whole. In the completed jigsaw puzzle, the fragments will form a complete picture of the red revolutionary ruins in the Soviet area of southern Jiangxi. On the virtual interactive contact, the user guide can trigger the event according to the direction of the character. For example, when virtual characters pass through the venue of the Soviet conference, it will trigger the playing of the "national anthem of the people's Republic of China" to enhance people's patriotism. On the whole, the red site of the Gannan Soviet area can let people deeply watch and understand the appearance of the revolutionary site of the whole Gannan Soviet area, and get an immersive experience in the visual user experience.

\section{Conclusion}

Red culture has become the precious wealth of Chinese spiritual culture for more than 70 years since the founding of the people's Republic of China. And the Red Revolution site in Gannan Soviet area is a kind of visible cultural carrier, which embodies the revolutionaries' hard work and plain living and not afraid of sacrifice. Experts and scholars in various fields have conducted in-depth analysis and research on the revolutionary sites in the Soviet area, and pointed out that the application of cutting-edge digital technology for the rescue and protection of the sites is of great significance. Therefore, we analyze it from the perspective of digital visualization. Under the platform of UE4 engine, we make full use of three-dimensional virtual simulation technology to model, material, light and render the red revolution site in the Soviet area, which can scientifically and effectively establish an interactive and visual digital scene experience. In the visual sense of the site, through the actual visual experience of the red revolution site in Gannan Soviet area, we can make the new generation of young people feel the inherent spiritual value of red culture closely and promote the development of red tourism in our country. On the other hand, the visualization of the Red Revolution site in the Soviet area will not only be a new way to protect the site, but also facilitate the propaganda and inheritance of the revolutionary site. On the whole, the red revolution sites in Gannan Soviet area are analyzed from a macro digital point of view in the study of key visualization technologies, which not only provides targeted technical thinking for the particularity of the sites, but also provides a guarantee for visualization effects. it also provides a reference for the development of other visualization projects.

\section{Acknowledgment}

Funds for the project: This paper is the phased achievement of the 2019 national college innovation and entrepreneurship of students training project:Research on Digital practice of Red Revolutionary sites in Soviet area based on VR Technology Virtual platform. The number of the project: 201910407033 .

\section{References}

1. Yang Rongbin. A Brief Discussion on the Site Management of the Memorial Hall of the Central Revolutionary Base in Ruijin [J]. Southern Cultural Relics, 2005 (6): 113,115.15.

2. A Study on the Value Utilization of the Former Site of the Revolution in Gannan Integrating into the Education of Secondary Vocational School Students[J]. Zhang Wenying. Art Science and Technology, 2018(06)

3. Gao Mingxing.Application of 3ds MAX Modeling and Scene Rendering Technology in Visual Design $[\mathrm{J}]$.Automation and Instrumentation, 2011 (6): 114-116.

4. Shi Min, Wang Junzheng et al. Realistic 3D Virtual Scene Construction and Roaming Method [J]. Journal of system Simulation, 2014 (9): 1969-1979

5. Yang Zhou. Architecture and Visual Design Method [J]. Chinese and Foreign Architecture, 2009 (1) 53-55.

6. Yang Shuguang. A Study on Visual Restoration of French Big Wood Technology Based on UE4 Engine [J]. Shanxi Architecture, 2019 (22): 5-8.

7. Shen Xiaoyun, Li Shuangxing, Jiao Weidong. "Design and Development of Digital Architecture Roaming Simulation Platform Based on Active X [J]. Journal of system Simulation, 2014 (1): 67-71.

8. Penning. Visual Representation in UI Design [J]. Electronic Technology and Software Engineering, 2020 (3): 101-103.

9. $\mathrm{Hu}$ Qiyun. An Analysis of Architectural Visualization of UE4 Game Engine [J]. Decoration, 2016 (8): 134,135.

10. Zhou Qiang, Zhang Minxiong, etc. Immersion Evaluation of Somatosensory Interactive Virtual Roaming [J]. Journal of Graphics, 2020(06) : 112-120. 